\title{
Practice Pearls for ReLEx SMILE in 2018
}

Dan Z Reinstein

DOl: https://doi.org/10.17925/EOR.2018.12.1.24

London Vision Clinic, London, UK; Department of Ophthalmology, Columbia University Medical Center, New York, NY, US; Centre Hospitalier

National d'Ophtalmologie, Paris, France; Biomedical Science Research Institute, University of UIster, Coleraine, UK

\section{Keywords}

Small incision lenticule extraction, SMILE, centration, recovery technique, docking, cap interface

Disclosure: Dan Z Reinstein is a consultant for Carl Zeiss Meditec (Jena, Germany), has a proprietary interest in the Artemis technology (ArcScan Inc, Morrison, Colorado) and is an author of patents related to VHF digital ultrasound administered by the Cornell Center for Technology

Enterprise and Commercialization (CCTEC), Ithaca, NY, US

Review Process: This article is a short educational piece and has not been submitted to external peer reviewers but was reviewed by the editorial board for accuracy before publication.

Authorship: All named authors meet the International Committee of Medical Journal Editors (ICMJE) criteria for authorship of this manuscript, take responsibility for the integrity of the work as a whole, and have given final approval to the version to be published.

open Access: This article is published under the Creative Commons Attribution Noncommercial License, which permits any noncommercial use, distribution, adaptation, and reproduction provided the original author(s) and source are given appropriate credit. (c) The Authors 2018.

Received: 18 May 2018

Published Online: 13 August 2018

Citation: European Ophthalmic Review. 2018;12(1):24-5

Corresponding Author: Dan Z Reinstein, London Vision Clinic, 138 Harley Street, London W1G 7LA, UK. E: dzr@londonvisionclinic.com

Support: No funding was received in the publication of this article.
Small incision lenticule extraction (SMILE) has been gaining major traction since it was fully commercialised in 2012, with more than 1.3 million procedures performed to date worldwide. Our first SMILE experiences began in 2010 during its development and since 2012 we have offered this routinely to our patients. On completion of our first 4,000 SMILE cases we performed a full data analysis of our evolving surgical technique, outcomes and complications which formed the basis for our recently published textbook 'The Surgeon's Guide to SMILE: Small Incision Lenticule Extraction'.' Over time we developed a fully standardised SMILE technique and a number of pearls that will help every physician ranging from a beginning novice to an expert who has performed thousands of cases. Here we share some insights that can improve outcomes as well as minimise and manage complications.

\section{Pearl 1 - Docking and centration}

In SMILE, the alignment of the refractive lenticule is effectively auto-centred by the patient fixating coaxially on a fixation light prior to suction being applied, resulting in lenticule formation centred on the corneal vertex of the coaxially fixating eye. No eye tracker is required during femtosecond cutting in SMILE as the eye is immobilised, although this means that if suction is applied incorrectly the treatment zone will be decentred. To help this process, we prepare a printout (Figure 1), including the topography eye image and the Hirschberg test, for the surgeon to compare to the intraoperative appearance. ${ }^{2}$

We find the topography eye image is the most useful reference for angle kappa and have developed a display that includes visual cues to quantify the magnitude and direction of any angle kappa (created by importing the Atlas image into PowerPoint and adding crosshairs; this template is available to download from www.londonvisionclinic.com/SMILECentrationTemplate). The docking process only takes a few seconds, so it is important for the surgeon to be able to immediately see, at a glance, the expected position of the reflex. However, the Atlas eye image is not infallible; for example, the patient may not have been coaxially fixating during acquisition, and the pupil diameter during treatment is usually slightly smaller than on the eye image, so these need to be mentally accounted for. With that being said, this is an easy double check to make sure the treatment is centred properly.

\section{Pearl 2 - Globe stabilisation during lenticule separation}

Many SMILE surgeons choose not to use an instrument to control the position of the eye during lenticule separation and many thousands of SMILE procedures have been done in this way. There is no reason to say that an immobilising instrument must be used, but the weight of advantages is with immobilisation and surgeon control of the globe. With the eye controlled by the surgeon, the force of the lenticule separation is controlled, whereas with a free-floating globe the eye will move around in the direction of the force applied with a force that is determined by the patient's extraocular muscle status. The use of a toothed-forceps at the limbus gripping down to Tenon's layer also allows the surgeon to apply counterforce against the lenticule separator.

Other disadvantages of allowing the globe to move freely with a single-instrument separation technique include the eye being shifted outside the surgical field of the microscope, producing a limited view or an increase in dissection time while the surgeon corrects the patient's fixation. 


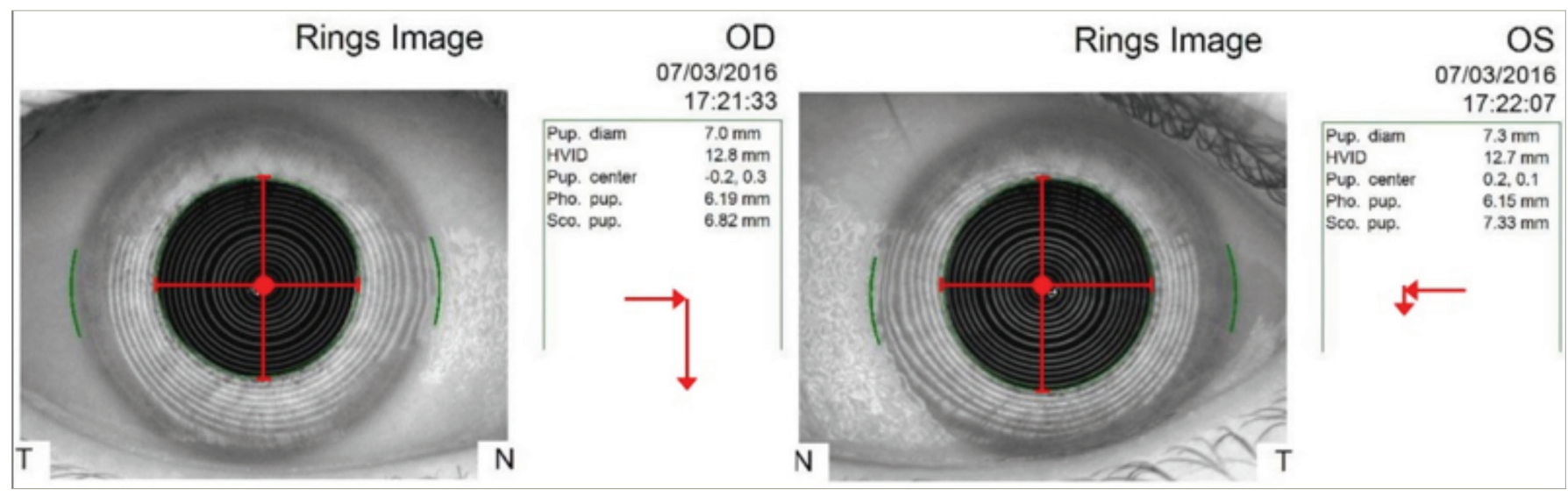

Reproduced with permission from Reinstein et al., $2018 .^{2}$

Figure 2: Cap interface recovery technique

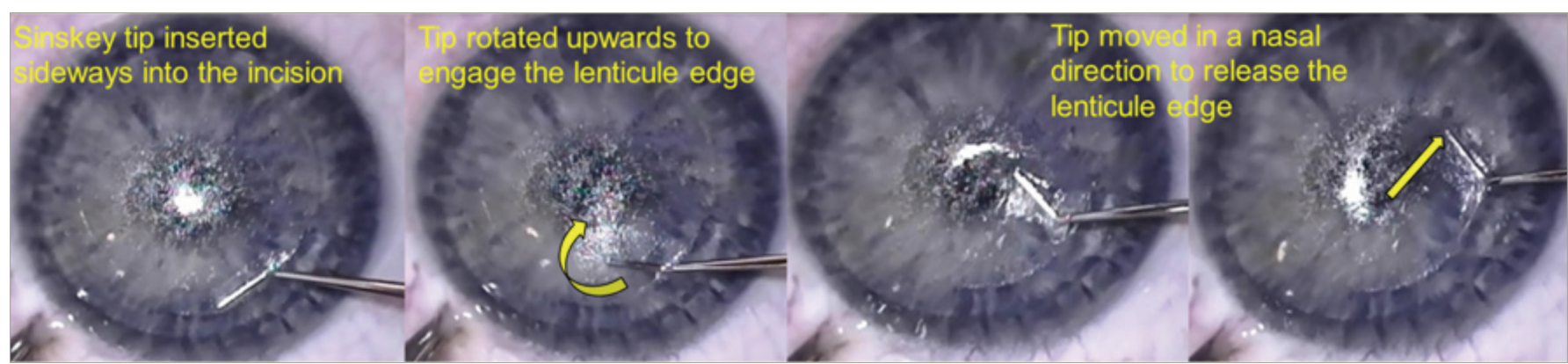

Reproduced with permission from Reinstein et al.,2018.4

Having a mobile eye makes it more difficult to follow a precise separation sequence, so you would expect there to be greater surgical manipulation required. A non-fixated eye also makes the surgery look more traumatic - purely from an aesthetic point of view - if you were to show a video to a patient, referring surgeon or optometrist, for example.

Our instrument of choice is a fine-toothed forceps. ${ }^{3}$ The forceps are used to hold and immobilise the globe during the interface separation part of the procedure. We elected to use toothed forceps over other blunt conjunctiva forceps as the globe needs to be held firmly enough to act against the force of separating the interfaces. The sharp teeth pierce through the conjunctiva and enable the surgeon to push down into Tenon's layer to provide a more stable grip. By holding the globe, the surgeon is able to apply counterforce against the interface separating instrument and enables more precise movements. Holding Tenon's as opposed to only conjunctiva (with conjunctival non-toothed forceps) produces conjunctival tears much less frequently.

\section{Pearl 3 - Lenticule from cap recovery technique}

Finally, one of the most important factors of which the surgeon should be aware is which interface was separated first. If this is known, then all your effort can be put into finding the other interface. Getting a feel for which interface you are in comes with experience - the most obvious cues include the visualisation of cap interface bubbles seen above the instrument, indicating you are in the lenticule interface; additionally, the cap interface extends to a larger diameter than the lenticule interface, which is visualised by the white ring appearance of the lenticule border. However, even experienced surgeons have cases where the lenticule interface is unintentionally separated first.

In this technique (Figure 2), the sinskey tip is inserted sideways into the small incision and moved across until the shaft reaches the edge limit of the incision. ${ }^{4}$ The tip is rotated upwards and anteriorly, tenting up the cap layer and engaging the lenticule edge in the process. This motion is successful the first time in virtually all cases, but may occasionally need to be repeated. Once the cap interface has been pocketed, the separation can proceed as normal. Armed with this technique, separating the lenticule interface first should no longer be considered a complication of SMILE and does not require any further special instrumentation.

SMILE is a user-friendly surgical option to treat refractive error. However, as with any other form of refractive surgery there is always room to improve the surgical technique and provide a smoother experience for all involved. We found these three pearls helped elevate confidence and improve outcomes and hope that other surgeons can benefit from our experience. $\square$

4. Chapter 10.1.1.2. Identification of cap interface if Ienticule interface was separated first. In: Reinstein DZ, Archer TA, Carp GI. The Surgeon's Guide to SMILE: Small Incision Lenticule Extraction. Thorofare, NJ: Slack Incorporated, 2018:238-40. 